\title{
New Medium for Selection and Presumptive Identification of the Bacteroides fragilis Group
}

\author{
SHIRLEY J. LIVINGSTON,' SPYROS D. KOMINOS, ${ }^{2}$ AND ROBERT B. YEE ${ }^{1 *}$ \\ Mercy Hospital, Pittsburgh, Pennsylvania 15219'; and Department of Microbiology, Graduate School of \\ Public Health, University of Pittsburgh, Pittsburgh, Pennsylvania 15261 ${ }^{1}$
}

Received for publication 28 September 1977

\begin{abstract}
A medium, Bacteroides fragilis bile-esculin (BBE) agar, was designed for the selection and presumptive identification of the $B$. fragilis group. BBE agar contains bile, esculin, ferric ammonium citrate, hemin, and gentamicin in a Trypticase soy agar base. Growth in the presence of $20 \%$ bile and esculin hydrolysis, detected by blackening of the medium, provide presumptive evidence for the identification of the $B$. fragilis group. In addition to stimulating the growth of many strains of the $B$. fragilis group, hemin provides the option of testing isolates for catalase production. Gentamicin and bile prevent the growth of most organisms other than the esculin-positive bacteroides that can tolerate bile. Of 160 clinical isolates of the $B$. fragilis group tested on BBE agar, 159 grew well on the medium and 157 blackened it. Other anaerobes, Enterobacteriaceae, and enterococci either failed to grow on $\mathrm{BBE}$ agar or did not produce the characteristic morphology and blackening associated with isolates of the $B$. fragilis group. In a clinical laboratory trial, 687 specimens from patients were inoculated onto $\mathrm{BBE}$ agar plates. The $B$. fragilis group was recovered from 81 (11.8\%) of these specimens in 24 to $48 \mathrm{~h}$. Use of BBE agar in the clinical laboratory enables earlier recovery and identification of this important pathogen.
\end{abstract}

Clinical laboratories should be able to quickly establish the presence of the Bacteroides fragilis group ( $B$. fragilis, $B$. diastonis, $B$. thetaiotaomicron, $B$. vulgatus) (14) in specimens from patients. Rapid identification of this pathogen plays an important role in determining the best chemotherapy for a patient. Unlike most anaerobes, members of the $B$. fragilis group are almost always resistant to penicillin (10-12) and can protect penicillin-sensitive bacteria accompanying them in mixed infections (13). In addition, this group of bacteria is the most frequently recovered anaerobe in the clinical laboratory (18), and thus efforts to simplify its recovery and identification can decrease the time and cost involved in anaerobic cultures.

Although advantageous, rapid recognition of the $B$. fragilis group presents several problems. (i) This pathogen must frequently be detected within and isolated from a complex mixture of facultative anaerobes and/or other anaerobes which can overgrow the primary plate $(6,8,21)$. (ii) Although other selective media rimplify the isolation of the $B$. fragilis group, they provide limited presumptive evidence for its identification (6), which means that all isolates must be subcultured and further tested. (iii) The conventional tests for the identification of the $B$. fragilis group may require $48 \mathrm{~h}$ or more for proper interpretation $(2,3)$.
In view of these problems, one approach for accelerating the recognition of the $B$. fragilis group has been the development of rapid identification tests which are based upon combinations of characteristics unique to this group. By one such test, any anaerobic gram-negative rod able to grow on tryptic soy agar with $20 \%$ bile and resistant to a $1,000-\mu \mathrm{g} / \mathrm{ml}$ kanamycin disk can be identified as a member of the $B$. fragilis group in $24 \mathrm{~h}(22)$. This test has been recently modified by using 25 -mg oxgall disks in conjunction with antibiotic identification disks (3). Both methods require prior isolation of the organisms in pure culture.

In this paper, we describe and evaluate a new primary plating medium, $B$. fragilis bile-esculin (BBE) agar, designed to select for the $B$. fragilis group and simultaneously provide presumptive evidence for its identification. This approach obviates the need for subculture and further testing and, therefore, reduces the total time required for recognition of the $B$. fragilis group to 24 to $48 \mathrm{~h}$.

(This paper was presented in part at the 77th Annual Meeting of the American Society for Microbiology, New Orleans, La., May 1977).

\section{MATERIALS AND METHODS}

Bacteria. The majority of anaerobes were recent clinical isolates from four hospitals in Pittsburgh, $\mathrm{Pa}$.: 
Mercy, Western Pennsylvania, Montefiore, and Presbyterian-University. The 63 clinical isolates included $8 \mathrm{~B}$. melaninogenicus, $1 \mathrm{~B}$. capillosus, $1 \mathrm{~B}$. oralis, 1 Fusobacterium nucleatum, 2 Fusobacterium sp., 4 Clostridium sporogenes, 3 C. perfringens, 1 C. clostridiiforme, $1 \mathrm{C}$. ghone, $1 \mathrm{C}$. irregularis, $1 \mathrm{C}$. sardiniensis, 1 C. tertium, 11 Peptococcus magnus, 7 P. prevotii, 5 P. asaccharolyticus, 1 Peptococcus sp., 8 Peptostreptococcus anaerobius, 2 P. micros, 1 Streptococcus morbillorum, 1 Veillonella alcalescens, $1 \mathrm{~V}$. parvula, and 1 Veillonella sp. In addition, 12 reference strains of anaerobes were obtained from the Center for Disease Control (CDC), Atlanta, Ga., and American Type Culture Collection (ATCC), Rockville, Md.: two strains of $F$. mortiferum (CDC 11747, ATCC 9817), two of $F$. varium (CDC 15749, ATCC 8501), two of $F$. necrophorum (CDC 18981, ATCC 25286), one of $F$. nucleatum (ATCC 25586), one of $B$. oralis (CDC 19270 ), one of $B$. melaninogenicus subsp. melaninogenicus (ATCC 25845), one of $B$. corrodens (CDC 19270), and two of $B$. melaninogenicus subsp. asaccharolyticus (B. asaccharolyticus) (CDC 19068, ATCC 25260). Facultative anaerobes were recent clinical isolates obtained from Mercy Hospital.

Stock cultures of anaerobes were maintained in tubes of cooked-meat medium (Difco Laboratories) and stored at room temperature in the dark. These cultures were transferred every 4 weeks and checked for contamination by streaking onto sheep blood agar (SBA) plates which were then incubated for $48 \mathrm{~h}$ at $36^{\circ} \mathrm{C}$ under aerobic conditions.

Isolates of the $B$. fragilis group were confirmed by testing for growth in $20 \%$ bile, esculin hydrolysis (2), catalase formation in the presence of hemin, and resistance to a $2-\mathrm{U}$ penicillin disk and a $1,000-\mu \mathrm{g} / \mathrm{ml}$ kanamycin disk (20). Catalase production was determined by exposing growth on BBE plates to the atmosphere for $0.5 \mathrm{~h}$ before adding $3 \%$ hydrogen peroxide, with a positive reaction being the evolution of bubbles. To identify the isolates by species, rhamnose, trehalose, and mannitol fermentation reactions and the indole test were used. Fermentation reactions were determined by CDC methods, which were modified by the addition of $1 \%$ bromothymol blue to the carbohydrate broths after adequate growth of the isolate had been obtained (2). The indole test was performed on 2-ml samples from 24 - to 48 -h cooked-meat-medium cultures (14).

Stock cultures of enterococci and Enterobacteriaceae were maintained on nutrient agar slants (Baltimore Biological Laboratory [BBL]) at room temperature. These cultures represented clinical isolates which had been identified by the microbiology laboratory at Mercy Hospital. Nevertheless, the cultures were tested by us in a minimal number of tests to confirm the results of the laboratory. The bile-esculin test on Enterococcosel agar (BBL) and growth in 6.5\% sodium chloride broth (5) were used to confirm isolates as enterococci. Enterobacteriaceae were identified by colony morphology on MacConkey agar (BBL) and by reactions on triple sugar iron agar $(\mathrm{BBL})$, lysine iron agar (BBL), urea agar (BBL), motility-indole-ornithine medium (Difco), and Simmon citrate agar (Difco) (4).

Media. BBE agar was prepared by dissolving $20 \mathrm{~g}$ of oxgall (Difco), $40 \mathrm{~g}$ of Trypticase soy agar (TSA)
(BBL), $1 \mathrm{~g}$ of esculin (Difco), and $0.5 \mathrm{~g}$ of ferric ammonium citrate (Fisher Scientific Co.) in 1 liter of distilled water with no heating required. A 2-ml amount of a $5-\mathrm{mg} / \mathrm{ml}$ stock hemin (Nutritional Biochemicals Corp.) solution and $2.5 \mathrm{ml}$ of a $40-\mathrm{mg} / \mathrm{ml}$ gentamicin solution (Garamycine injectable, Schering Laboratories) were then added, and the $\mathrm{pH}$ was adjusted to 7.0. The medium was autoclaved for $15 \mathrm{~min}$ at $121^{\circ} \mathrm{C}$.

The $5-\mathrm{mg} / \mathrm{ml}$ stock hemin solution was prepared by dissolving $0.5 \mathrm{~g}$ of hemin in $10 \mathrm{ml}$ of $1 \mathrm{~N}$ sodium hydroxide and bringing the volume to $100 \mathrm{ml}$ with distilled water. The solution was autoclaved at $121^{\circ} \mathrm{C}$ for $15 \mathrm{~min}$ and stored at $4^{\circ} \mathrm{C}$.

SBA plates were prepared by adding defibrinated sheep blood (Sack's Farms, Evans City, Pa.) to autoclaved TSA base to give a final concentration of $5 \%$ or were obtained from BBL. For more fastidious anaerobes, SBA was supplemented with $5 \mu \mathrm{g}$ of hemin per $\mathrm{ml}$ and $10 \mu \mathrm{g}$ of vitamin $\mathrm{K}_{1}$ (Nutritional Biochemicals) per ml (21). Kanamycin-vancomycin-laked blood agar plates (KV) were obtained from BBL.

Culturing methods. Samples of 48-h cookedmeat-medium cultures of the $B$. fragilis group were aseptically removed and placed in sterile tubes. Their turbidity was adjusted with sterile distilled water to match that of a $0.5 \mathrm{McF}$ arland standard. After being blended in a Vortex mixer, these suspensions were used to inoculate BBE and SBA plates, $0.01 \mathrm{ml}$ of inoculum per plate. The plates were then streaked in a conventional four-quadrant pattern with a sterile 3mm-diameter loop. Flaming of the loop between quadrants was not done. After incubation in GasPak jars (BBL) for $48 \mathrm{~h}$ at $36^{\circ} \mathrm{C}$, growth on each plate was graded as 0 (no growth), $1+$ (growth in the first quadrant), 2+ (growth in the first two quadrants), 3+ (growth in the first three quadrants), and 4+ (growth in all four quadrants). A strain was considered inhibited if growth on the BBE plate was more than two grades below that on the SBA plate.

Other anaerobes were cultured in a similar manner except each strain was inoculated on a single quadrant of a plate, and supplemented SBA plates were used for more fastidious bacteria.

Each facultative anaerobe was cultured by using a calibrated loop to inoculate a quadrant of each of a BBE, KV, and SBA (for swarming Proteus sp., MacConkey agar was substituted) plate with $0.001 \mathrm{ml}$ of a 4-h Trypticase soy broth culture. Results were recorded as growth (presence of macroscopic colonies) or no growth after $48 \mathrm{~h}$ of anaerobic incubation.

Clinical laboratory trial. A 4-month field study was established in the clinical microbiology laboratory at Mercy Hospital. Technicians were instructed to plant the following types of specimens on a KV plate and one-half of a BBE plate: wounds and drainages; abscesses, ulcers, and decubiti; body fluids and exudates; materials from biopsies and autopsies and cervical-uterine swabs from in-house patients only. After smears were prepared for Gram staining, the media were inoculated in the following order: thioglycolate broth, aerobic and anaerobic SBA, KV agar, BBE agar, and others. KV plates were stored in holding jars through which $\mathrm{CO}_{2}$ was flowing to partially protect the medium from oxidation. Due to lack of space, BBE plates were not stored in holding jars. All plates were 
incubated in GasPak jars at $36^{\circ} \mathrm{C}$ and were examined at $24 \mathrm{~h}$, with the final results being recorded at $48 \mathrm{~h}$. All colonies growing on $\mathrm{KV}$ agar and $\mathrm{BBE}$ agar were subcultured and identified with conventional procedures.

\section{RESULTS}

A total of 160 clinical isolates of the $B$. fragilis group were tested for their ability to grow on BBE agar and to blacken the medium, evidence for esculin hydrolysis (Table 1). Only one isolate previously identified as $B$. fragilis failed to grow on the medium. The use of a conventional tube test confirmed that this strain was inhibited by $20 \%$ bile and, therefore, should be considered an atypical strain or Bacteroides sp. Of 159 isolates which grew on BBE agar, only 2 failed to blacken the medium. These isolates, identified as $B$. vulgatus, did not hydrolyze esculin by the conventional broth method, and thus their inability to darken the medium was not an unexpected finding.

In addition, all isolates of the $B$. fragilis group which grew on BBE agar were tested for catalase production, with $97 \%$ being positive for this characteristic. Seven isolates, one of $B$. thetaiotaomicron, one of $B$. distasonis, and five of $B$. vulgatus failed to produce catalase on BBE agar.

The isolates of the $B$. fragilis group produced three types of colonies on BBE agar. All three types of colonies appeared circular, entire, raised, and greater than $1 \mathrm{~mm}$ in diameter after $48 \mathrm{~h}$ of anaerobic incubation. Colonies designated as type 1 were dull, opaque, brittle, charcoal gray, and surrounded by a dark-gray zone and precipitate. In contrast, type 2 colonies were glistening, semitranslucent, butyrous, light-todark gray, and surrounded by a gray zone with no precipitate. Isolates that were unable to hydrolyze esculin produced type 3 colonies, which resembled those of type 2 but were white and lacked gray zones. A total of $31 \%$ of the isolates yielded type 1 colonies, with $94 \%$ of these being identified as $B$. fragilis; $68 \%$ produced type 2 colonies; and $1 \%$ produced type 3 .

A total of 63 clinical isolates and the 12 reference strains of anaerobes other than members of the $B$. fragilis group were tested on BBE agar (Table 2). Only four of these anaerobes, the reference strains of $F$. mortiferum and $F$. varium, grew on the medium. This observation could be predicted because these fusobacteria are resistant to $20 \%$ bile (19). However, the colonies of the four strains were "fried egg" in appearance and, with the exception of $F$. mortiferum strain CDC 11747, were less than $1 \mathrm{~mm}$ in size due to their relatively greater sensitivity to gentamicin (7). The colony produced by this CDC reference strain, which could hydrolyze esculin by the conventional test, possessed a
TABLE 1. Growth and blackening produced by clinical isolates of the $B$. fragilis group on $B B E$ agar $^{a}$

\begin{tabular}{lccc}
\hline \multirow{2}{*}{ B. fragilis group } & $\begin{array}{c}\text { No. of iso- } \\
\text { lates } \\
\text { tested }\end{array}$ & BBE agar \\
\cline { 3 - 4 } & 104 & 103 & 103 \\
\hline B. fragilis & 35 & 35 & 35 \\
B. thetaiotaomicron & 13 & 13 & 13 \\
B. distasonis & 7 & 7 & 5 \\
B. vulgatus & 1 & 1 & 1 \\
B. ovatus & 160 & 159 & 157 \\
\multicolumn{1}{c}{ Total } & & & $\begin{array}{c}\text { Blacken- } \\
\text { ing }\end{array}$ \\
\hline
\end{tabular}

" Results were recorded after $48 \mathrm{~h}$ of anaerobic incubation

${ }^{\circ}$ All isolates grew on control plates of SBA.

TABLE 2. Growth and blackening produced by anaerobes (other than the $B$. fragilis group) on $B B E$ agar"

\begin{tabular}{|c|c|c|c|}
\hline \multirow[b]{2}{*}{ Organism } & \multirow{2}{*}{$\begin{array}{c}\text { No. of iso- } \\
\text { lates and } \\
\text { strains } \\
\text { tested }^{h}\end{array}$} & \multicolumn{2}{|c|}{ BBE agar } \\
\hline & & Growth & $\begin{array}{l}\text { Blacken- } \\
\text { ing }\end{array}$ \\
\hline Bacteroides sp. & 15 & 0 & 0 \\
\hline Fusobacterium sp. & 10 & 4 & $1^{\prime}$ \\
\hline Clostridium sp. & 12 & 0 & 0 \\
\hline Peptococcus sp. & 24 & 0 & 0 \\
\hline Peptostreptococcus sp. & 10 & 0 & 0 \\
\hline Streptococcus sp. & 1 & 0 & 0 \\
\hline Veillonella sp. & 3 & 0 & 0 \\
\hline Total & 75 & 4 & 1 \\
\hline
\end{tabular}

"Results were recorded after $48 \mathrm{~h}$ of anaerobic incubation at $36^{\circ} \mathrm{C}$.

${ }^{h}$ All anaerobes grew on control plates of SBA or supplemented SBA.

'Colony morphology differed from that of the $B$. fragilis group.

light-gray center but lacked a surrounding gray zone. In addition, all four strains failed to produce catalase on BBE agar.

A total of 118 clinical isolates of facultative anaerobes were tested on $\mathrm{BBE}$ agar and $\mathrm{KV}$ agar under anaerobic conditions (Table 3). Thirteen of the isolates grew on BBE agar and only one of the organisms, Klebsiella pneumoniae, was able to blacken the medium. The colonies of all of these 13 isolates could be readily distinguished from those of $B$. fragilis. After $48 \mathrm{~h}$ of anaerobic incubation, the strain of $K$. pneumoniae produced pinpoint colonies that were brownish-black rather than gray. The other isolates yielded flat, transparent or translucent colonies, often with an irregular edge and usually less than $1 \mathrm{~mm}$ in diameter. $\mathrm{KV}$ agar permitted the growth of 28 facultative anaerobes.

In the clinical laboratory trial, 687 specimens were inoculated on both BBE agar and $\mathrm{KV}$ agar. A total of $81(11.8 \%)$ of the specimens yielded the $B$. fragilis group on BBE agar, whereas 75 $(10.9 \%)$ were positive on $\mathrm{KV}$ agar. Six more 
specimens were positive for the $B$. fragilis group on $\mathrm{BBE}$ agar than on $\mathrm{KV}$ agar, a difference that was not statistically significant at $\alpha=0.01$ using the chi-square test (9).

A total of 95 isolates of the $B$. fragilis group, with $B$. fragilis predominating, were recovered on BBE agar during the clinical trial (Table 4). All of these isolates except two, one of $B$. fragilis and one of $\boldsymbol{B}$. distasonis, were recovered as gray colonies on BBE agar and could have been presumptively identified as members of the $B$. fragilis group at $48 \mathrm{~h}$ from the primary plates. When the inoculum was sufficient, growth and some darkening of the medium could be detected by $24 \mathrm{~h}$.

During the clinical laboratory trial, organisms other than those of the $B$. fragilis group were isolated on BBE agar and KV agar (Tables 5 and 6). KV agar recovered 30 isolates of anaerobic, gram-negative rods other than the $B$. fragilis group, none of which grew on BBE agar. In addition, KV agar permitted the growth of 36 facultative anaerobic gram-negative rods. These included two strains of Proteus mirabilis, which swarmed over the primary plate, a problem not encountered with BBE agar. Only three isolates of Providencia sp. and two of Proteus sp. were

TABLE 3. Comparison of growth inhibition of facultative anaerobes on $K V$ agar and BBE agar

\begin{tabular}{|c|c|c|c|c|}
\hline \multirow{2}{*}{ Organism } & \multirow{2}{*}{$\begin{array}{l}\text { No. of iso- } \\
\text { lates } \\
\text { tested }^{b}\end{array}$} & \multirow{2}{*}{$\frac{\text { KV agar }}{\text { Growth }}$} & \multicolumn{2}{|c|}{ BBE agar } \\
\hline & & & Growth & $\begin{array}{l}\text { Blacken- } \\
\text { ing }\end{array}$ \\
\hline Escherichia coli & 14 & 2 & 0 & 0 \\
\hline $\begin{array}{l}\text { Citrobacter freun- } \\
\text { dii }\end{array}$ & 5 & 1 & 1 & 0 \\
\hline C. diversus & 4 & 0 & 0 & 0 \\
\hline $\begin{array}{l}\text { Serratia marces- } \\
\text { cens }\end{array}$ & 13 & 7 & 0 & 0 \\
\hline S. rubidaea & 1 & 0 & 0 & $\mathbf{0}$ \\
\hline S. liquefaciens & 1 & 0 & $\mathbf{0}$ & $\mathbf{0}$ \\
\hline $\begin{array}{c}\text { Klebsiella pneu- } \\
\text { moniae }\end{array}$ & 13 & 2 & 1 & $1^{c}$ \\
\hline $\begin{array}{c}\text { Enterobacter aer } \\
\text { ogenes }\end{array}$ & 8 & 0 & 0 & 0 \\
\hline E. cloacae & 7 & 1 & 0 & $\mathbf{0}$ \\
\hline E. agglomerans & 2 & 0 & $\mathbf{0}$ & 0 \\
\hline E. hafniae & 1 & 0 & 0 & 0 \\
\hline Providencia sp. & 7 & 6 & 6 & 0 \\
\hline Proteus mirabilis & 5 & 4 & 0 & 0 \\
\hline$P$. rettgeri & 5 & 5 & 4 & 0 \\
\hline$P$. vulgaris & 1 & 0 & 0 & $\mathbf{0}$ \\
\hline P. morganii & 1 & 0 & 1 & $\mathbf{0}$ \\
\hline Enterococci & 30 & 0 & 0 & 0 \\
\hline Total & 118 & 28 & 13 & 1 \\
\hline
\end{tabular}

${ }^{a}$ Results were recorded after $48 \mathrm{~h}$ of anaerobic incubation at $36^{\circ} \mathrm{C}$.

${ }^{b}$ All isolates grew on control plates of SBA or MacConkey agar.

"Colony morphology differed from that of the $B$. fragilis group.
TABLE 4. Recovery of the B. fragilis group on BBE agar during clinical laboratory trial ${ }^{a}$

\begin{tabular}{|c|c|}
\hline B. fragilis & $\begin{array}{c}\text { No. of isolates re- } \\
\text { covered }\end{array}$ \\
\hline B. fragilis & 67 \\
\hline B. thetaiotaomicron & 17 \\
\hline B. distasonis . . . . & 8 \\
\hline B. vulgatus & 2 \\
\hline Total & 94 \\
\hline
\end{tabular}

a The $B$. fragilis group was recovered from $81 \mathrm{spec}-$ imens (11.8\%) of 687 specimens cultured. Some specimens yielded more than one species.

TABLE 5. Comparison of recovery of gram-negative rods other than the $B$. fragilis group on $K V$ agar and BBE agar from 687 clinical specimens $^{a}$

\begin{tabular}{lrrc}
\hline \multirow{2}{*}{\multicolumn{1}{c}{ Gram-negative rod }} & \multicolumn{3}{c}{ Growth on } \\
\cline { 2 - 4 } & SBA & $\begin{array}{c}\text { KV } \\
\text { agar }\end{array}$ & $\begin{array}{c}\text { BBE } \\
\text { agar }^{b}\end{array}$ \\
\hline Escherchia coli & 98 & 10 & 0 \\
Enterobacter cloacae & 13 & 2 & 0 \\
Klebsiella pneumoniae & 45 & 7 & 0 \\
Providencia sp. & 12 & 11 & 3 \\
Proteus rettgeri & 3 & 2 & 1 \\
$P$. morganii & 4 & 2 & 1 \\
$P$. mirabilis & 31 & 2 & 0 \\
Anaerobic gram-negative & 30 & 30 & 0 \\
$\quad$ rods & & & \\
$\quad$ Total & 236 & 66 & 5 \\
\hline
\end{tabular}

${ }^{a}$ Results after $48 \mathrm{~h}$ of anaerobic incubation at $36^{\circ} \mathrm{C}$.

${ }^{b}$ None of the isolates blackened the medium.

TABLE 6. Comparison of recovery of organisms other than gram-negative rods on $K V$ agar and $B B E$ agar from 687 clinical specimens ${ }^{a}$

\begin{tabular}{lrrr}
\hline \multirow{2}{*}{ Organism } & \multicolumn{3}{c}{ Growth on } \\
\cline { 2 - 4 } & SBA & $\begin{array}{c}\text { KV } \\
\text { agar }\end{array}$ & $\begin{array}{c}\text { BBE } \\
\text { agar }^{h}\end{array}$ \\
\hline Staphylococcus epidermidis & 113 & 7 & 1 \\
S. aureus & 108 & 1 & 0 \\
Enterococci & 47 & 1 & 0 \\
Diphtheroids & 32 & 1 & 1 \\
Yeast & 18 & 18 & 18 \\
Veillonella sp. & 2 & 0 & 0 \\
$\quad$ & 320 & 28 & 20 \\
\hline \multicolumn{1}{l}{ Total } & &
\end{tabular}

${ }^{a}$ Results were recorded after $48 \mathrm{~h}$ of anaerobic incubation at $36^{\circ} \mathrm{C}$.

${ }^{b}$ None of these isolates blackened the medium.

recovered on BBE agar, and none of these blackened the medium. Both media failed to inhibit yeast.

In a limited study, five isolates of the $B$. fragilis group and 20 facultative anaerobes were tested on freshly poured BBE plates and on plates from the same batch that had been stored 
for 6 weeks at $4^{\circ} \mathrm{C}$. Refrigerated storage did not affect the ability of BBE agar to support the growth of isolates of the $B$. fragilis group or to inhibit the facultative anaerobes.

\section{DISCUSSION}

Because members of the $B$. fragilis group are almost always resistant to penicillin, we sought to develop a selective and differential medium for this pathogen and thereby reduce the total time required for recognition of the $B$. fragilis group in clinical specmens. The study with stock cultures of clinical isolates and the clinical laboratory trial indicates that BBE agar meets these objectives. While supporting the growth of the majority of strains of the $B$. fragilis group, BBE agar inhibits almost all other anaerobes and is twice as efficient as KV agar in suppressing facultative anaerobes. In addition, isolates of the $B$. fragilis group yield colonies on BBE agar which are easily recognized and differentiated from those of other bacteria, and, thus, tentative identification can be made from the primary plate within $48 \mathrm{~h}$.

The use of BBE agar presents several other advantages to the clinical laboratory. (i) Its high selectivity decreases the number of unnecessary subcultures and the cost of adding a new medium by permitting the use of halves or quadrants of plates. (ii) Identification of the $B$. fragilis group directly from the primary plate eliminates the cost of additional media that would otherwise be required for the subculture and identification of an isolate. (iii) $\mathrm{BBE}$ agar can be employed as a differential medium for the subculture of positive anaerobic blood bottles and other broths. (iv) A limited study suggests that BBE agar is stable enough to be prepared in large batches and stored at $4^{\circ} \mathrm{C}$ for 3 to 6 weeks without loss in ability to support the growth of the $B$. fragilis group or to inhibit facultative anaerobes. (v) The simplicity of its formula makes BBE agar easy to prepare from ingredients available to most clinical laboratories.

An examination of those ingredients which compose BBE agar indicates how this medium functions successfully. BBE agar consists of gentamicin, oxgall, hemin, esculin, and ferric ammonium citrate in a TSA base. Selective inhibition is accomplished by means of gentamicin, a broad-spectrum antibiotic, and oxgall. The gentamicin, at a concentration of $100 \mu \mathrm{g} / \mathrm{ml}$, suppresses facultative anaerobes while permitting the $B$. fragilis group to grow because most strains of this pathogen have minimal inhibitory concentrations of $800 \mu \mathrm{g} / \mathrm{ml}$ or greater (7). In addition, gentamicin, due to its heat stability, does not lose its activity at incubator tempera- tures and can be incorporated into BBE agar before sterilization (1). The oxgall inhibits almost all anaerobic gram-negative rods except the $B$. fragilis group (18). In addition to acting as a required growth factor for many strains of the $B$. fragilis group (23), hemin provides the option of testing isolates for catalase production (T. D. Wilkins et al., Abstr. Ann. Meet. Am. Soc. Microbiol., 1977, C173, p. 64). Esculin hydrolysis is detected by a reaction between ferric ammonium citrate and esculetin, a breakdown product, to form a black complex (16). Members of the $B$. fragilis group produce gray colonies on BBE agar because of their ability to grow on this medium and hydrolyze esculin. Catalase is also produced.

In summary, $B$. fragilis bile-esculin or $\mathrm{BBE}$ agar is a new selective and differential medium for the $B$. fragilis group which enables earlier recovery and identification of this important pathogen. We caution that this medium will not permit the detection of all penicillin-resistant bacteroides, namely, those which are inhibited by bile. For example, B. melaninogenicus and $B$. oralis failed to grow on the medium. Penicillin-resistant strains of these bacteroides, $B$. $d i$ siens and $B$. bivius, have been described by other workers $(15,17)$.

\section{ADDENDUM}

Subsequent to the submission of this manuscript, comparable results on a similar medium were reported by P. C. K. Chan and R. K. Porschen, 1977, J. Clin. Microbiol. 6:528-529.

\section{ACKNOWLEDGMENTS}

We thank George Lombard for supplying CDC reference strains of anaerobes, A. William Pasculle and Patricia Carothers for assisting in the preparation of this manuscript, Robert Wadowsky for isolates of Enterobacteriaceae, and Carol Delenko and Dolores Freedel for their invaluable advice and encouragement. We are especially indebted to the many individuals who compose the microbiology staffs of Mercy, Western Pennsylvania, Presbyterian-University, and Montefiore Hospitals.

This study was supported by Public Health Service grant no. 5 D04 AH01437, and SJL received traineeship support from grant no. 1 A04 AH00913. Both grants were from the Bureau of Health Manpower, Division of Allied Health Professions, U.S. Public Health Service.

\section{LTERATURE CITED}

1. Casemore, D. P. 1967. Gentamicin as a bactericidal agent in virological tissue cultures. J. Clin. Pathol. 20:298-299.

2. Dowell, V. R., Jr., and T. M. Hawkins. 1974. Laboratory methods in anaerobic bacteriology. CDC laboratory manual. U.S. Department of Health, Education and Welfare, Washington, D.C.

3. Draper, D. L., and A. L. Barry. 1977. Rapid identification of Bacteroides fragilis with bile and antibiotic disks. J. Clin. Microbiol. 5:439-443.

4. Edwards, P. R., and W. H. Ewing. 1972. Identification of Enterobacteriaceae, 3rd ed. Burgess Publishing Co., Minneapolis.

5. Facklam, R. R. 1974. Streptococci, p. 96-108. In E. H. 
Lennette, E. H. Spaulding, and J. P. Truant (ed.), Manual of clinical microbiology, 2nd ed. American Society for Microbiology, Washington, D.C.

6. Finegold, S. M., P. T. Sugihara, and V. L. Sutter. 1971. Use of selective media for the isolation of anaerobes from humans, p. 99-108. In D. A. Shapton and R. G. Board (ed.), Isolation of anaerobes. Cox and Wyman Ltd., Norfolk, England.

7. Finegold, S. M., and V. L. Sutter. 1971. Susceptibility of gram-negative anaerobic bacilli to gentamicin and other aminoglycosides. J. Infect. Dis. 124:556-558.

8. Finegold, S. M., V. L. Sutter, H. R. Attebery, and J. E. Rosenblatt. 1974. Isolation of anaerobic bacteria, $p$. 365-375. In E. H. Lennette, E. H. Spaulding, and J. P. Truant (ed.), Manual of clinical microbiology, 2nd ed. American Society for Microbiology, Washington, D.C.

9. Freund, J. E. 1967. Modern elementary statistics, 3rd ed. p. 272-277. Prentice-Hall, Inc., Englewood Cliffs, N.J.

10. Gorbach, S. L., and J. G. Bartlett. 1974. Medical progress: anaerobic infections. N. Engl. J. Med. 290:1177-1184.

11. Gorbach, S. L., and J. G. Bartlett. 1974. Medical progress: anaerobic infections. N. Engl. J. Med. 290: $1237-1245$

12. Gorbach, S. L., and J. G. Bartlett. 1974. Medical prog ress: anaerobic infections. N. Engl. J. Med. 290:1289-1294.

13. Hackman, A. S., and T. D. Wilkins. 1975. In vivo protection of Fusobacterium necrophorum from penicillin by Bacteroides fragilis. Antimicrob. Agents Chemother. 7:698-703.

14. Holdeman, L. V., E. P. Cato, and W. E. C. Moore (ed.). 1977. Anaerobe laboratory manual, 4th ed. Virginia Polytechnic Institute and State University, Blacksburg.

15. Holdeman, L. V., and J. L. Johnson. 1977. Bacteroides disiens sp. nov. and Bacteroides bivius sp. nov. from human clinical infections. J. Clin. Microbiol. 6:337-345.

16. MacFaddin, J. F. 1976. Biochemical tests for identification of medical bacteria, p. 4-8. The Williams \& Wilkins Co., Baltimore.

17. Salyers, A. A., J. Wong, and T. D. Wilkins. 1977. Betalactamase activity in strains of Bacteroides melaninogenicus and Bacteroides oralis. Antimicrob. Agents Chemother. 11:142-146.

18. Shimada, K., V. L. Sutter, and S. M. Finegold. 1970. Effect of bile and desoxycholate on gram-negative anaerobic bacteria. Appl. Microbiol. 20:737-741.

19. Sutter, V. L., H. R. Attebery, and S. M. Finegold. 1974. Gram-negative nonsporeforming anaerobic bacilli, p. 388-395. In E. H. Lennette, E. H. Spaulding, and J. P. Truant (ed.), Manual of clinical microbiology, 2nd ed. American Society for Microbiology, Washington, D.C.

20. Sutter, V. L., and S. M. Finegold. 1971. Antibiotic disc susceptibility tests for rapid presumptive identification of gram-negative anaerobic bacilli. Appl. Microbiol. 21:13-20.

21. Sutter, V. L., V. L. Vargo, and S. M. Finegold. 1975. Wadsworth anaerobic bacteriology manual, 2nd ed. University of California, Los Angeles, Extension Division, Los Angeles.

22. Vargo, V., M. Korzeniowski, and E. H. Spaulding. 1974. Tryptic soy bile-kanamycin test for identification of Bacteroides fragilis. Appl. Microbiol. 27:480-483.

23. Wilkins, T. D., S. L. Chalgren, F. Jimenez-Ulate, C. R. Drake, Jr., and J. L. Johnson. 1976. Inhibition of Bacteroides fragilis on blood agar plates and reversal of inhibition by added hemin. J. Clin. Microbiol. $3: 359-363$. 